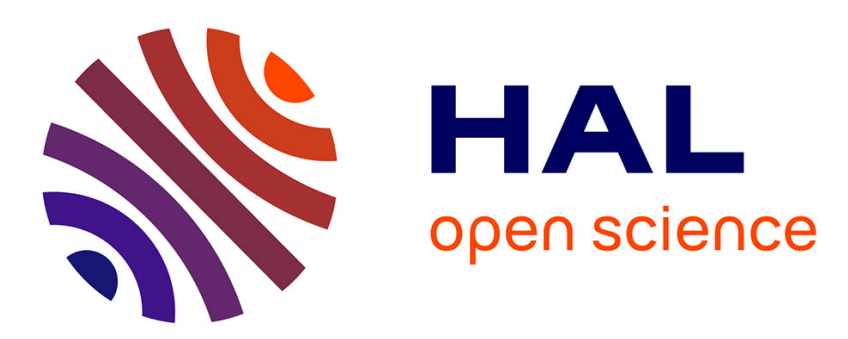

\title{
Influence of third-order dispersion on the propagation of incoherent light in optical fibers
}

Christine Michel, P. Suret, S. Randoux, Hans-Rudolf Jauslin, Antonio Picozzi

\section{To cite this version:}

Christine Michel, P. Suret, S. Randoux, Hans-Rudolf Jauslin, Antonio Picozzi. Influence of third-order dispersion on the propagation of incoherent light in optical fibers. Optics Letters, 2010, 35, pp.2367. hal-00696676

\section{HAL Id: hal-00696676 \\ https://hal.science/hal-00696676}

Submitted on 13 May 2012

HAL is a multi-disciplinary open access archive for the deposit and dissemination of scientific research documents, whether they are published or not. The documents may come from teaching and research institutions in France or abroad, or from public or private research centers.
L'archive ouverte pluridisciplinaire HAL, est destinée au dépôt et à la diffusion de documents scientifiques de niveau recherche, publiés ou non, émanant des établissements d'enseignement et de recherche français ou étrangers, des laboratoires publics ou privés. 


\title{
Influence of third-order dispersion on the propagation of incoherent light in optical fibers
}

\author{
C. Michel, ${ }^{1, *}$ P. Suret, ${ }^{2}$ S. Randoux,${ }^{2}$ H. R. Jauslin, ${ }^{1}$ and A. Picozzi ${ }^{1}$ \\ ${ }^{1}$ Laboratoire Interdisciplinaire Carnot de Bourgogne, UMR-CNRS 5209, Université de Bourgogne, France \\ ${ }^{2}$ Laboratoire de Physique des Lasers, Atomes et Molecules, UMR-CNRS 8523, Université de Lille, France \\ *Corresponding author: claire.michel@u-bourgogne.fr
}

Received April 2, 2010; revised June 3, 2010; accepted June 3, 2010; posted June 21, 2010 (Doc. ID 126414); published July 7, 2010

\begin{abstract}
We study the influence of third-order dispersion effects on the propagation of an incoherent nonlinear wave in an optical fiber system. The wave spectrum is shown to exhibit a highly asymmetric deformation characterized by a lateral spectral shoulder and the subsequent formation of an unexpected constant spectral pedestal. A kinetic approach to the problem reveals the existence of an invariant that explains in detail the essential properties of such asymmetric spectral evolution of the wave. () 2010 Optical Society of America

OCIS codes: $190.4370,190.4380,030.1640$.
\end{abstract}

The analysis of the long-term evolution of an incoherent nonlinear optical field is attracting growing interest [1-ㅛ] In particular, the thermalization of an optical wave has been studied in various circumstances, such as supercontinuum generation $[\underline{9}, 10]$ or spontaneous repolarization [11], as well as in various optical media characterized by different nonlinearities $[\underline{5}, \underline{7}, \underline{12}, \underline{13}]$. In complete analogy with the kinetics of a gas system, optical wave thermalization manifests itself by means of an irreversible evolution of the optical field toward the thermodynamic equilibrium state, i.e., the Rayleigh-Jeans (RJ) spectrum. Wave turbulence (WT) theory [14] is known to provide a detailed description of this nonequilibrium thermalization process. In a recent work, we identified a process of anomalous thermalization [15] that originates in degenerate resonances of the wave system. It is characterized by an irreversible evolution of the wave toward a local equilibrium state that violates the property of energy equipartition inherent to the RJ spectrum.

Our aim here is to address the consequences of this anomalous thermalization in an optical system of practical interest. We consider the problem of the propagation of an incoherent wave in an optical fiber near by the zero dispersion wavelength. In spite of its simplicity and its natural fundamental importance, this problem has not been treated so far, to the best of our knowledge. We show that the perturbative third-order dispersion (TOD) effect is responsible for a highly asymmetric deformation of the spectrum, which may be described in detail by the WT theory: Following the methodology developed in [15], we derive a kinetic equation that describes the spectral evolution of the wave in quantitative agreement with the numerical simulations of the nonlinear Schrödinger equation (NLSE). Furthermore, the kinetic approach reveals the existence of a rather simple conserved quantity (invariant), which explains all the essential properties of the complex evolution of the wave spectrum.

We consider the scalar NLSE in the presence of TOD:

$$
i \partial_{z} u=-\sigma \partial_{t}^{2} u+i \alpha \partial_{t}^{3} u+|u|^{2} u .
$$

We normalized the problem with respect to the nonlinear length $L_{0}=1 / \gamma P$ and time $\tau_{0}=\left(\left|\beta_{2}\right| L_{0} / 2\right)^{1 / 2}$, where $\gamma$ is the nonlinear coefficient, $P$ is the average power of the wave, and $\beta_{2}$ is the second-order dispersion coefficient. In these units, the normalized TOD parameter reads $\alpha=\beta_{3} /\left(6 L_{0}^{1 / 2}\left(\left|\beta_{2}\right| / 2\right)^{3 / 2}\right)$, with $\beta_{3}$ as the TOD coefficient and $\sigma=\operatorname{sign}\left(\beta_{2}\right)$. The NLSE conserves three important quantities: the normalized power $N=\int|u(t)|^{2} d t$, the momentum $M=\int \omega|\tilde{u}(\omega)|^{2} d \omega$, and the "energy" (Hamiltonian) $H=E+U$, which has a linear (dispersive) kinetic contribution $E=\int k(\omega)|\tilde{u}(\omega)|^{2} d \omega$ and a nonlinear contribution $U=\frac{1}{2} \int|u(t)|^{4} d t$, where $k(\omega)=\sigma \omega^{2}+\alpha \omega^{3}$ is the linear dispersion relation and $\tilde{u}(z, \omega)=\frac{1}{\sqrt{2 \pi}} \int u(z, t)$ $\exp (-i \omega t) d t$ is the Fourier transform of $u(z, t)$. The zero dispersion frequency is $\omega_{0}=-\sigma /(3 \alpha)$. We recall that TOD breaks the integrability of the scalar NLSE.

Figure 1 illustrates the first stage of the typical evolution of the spectrum of the field obtained by integrating
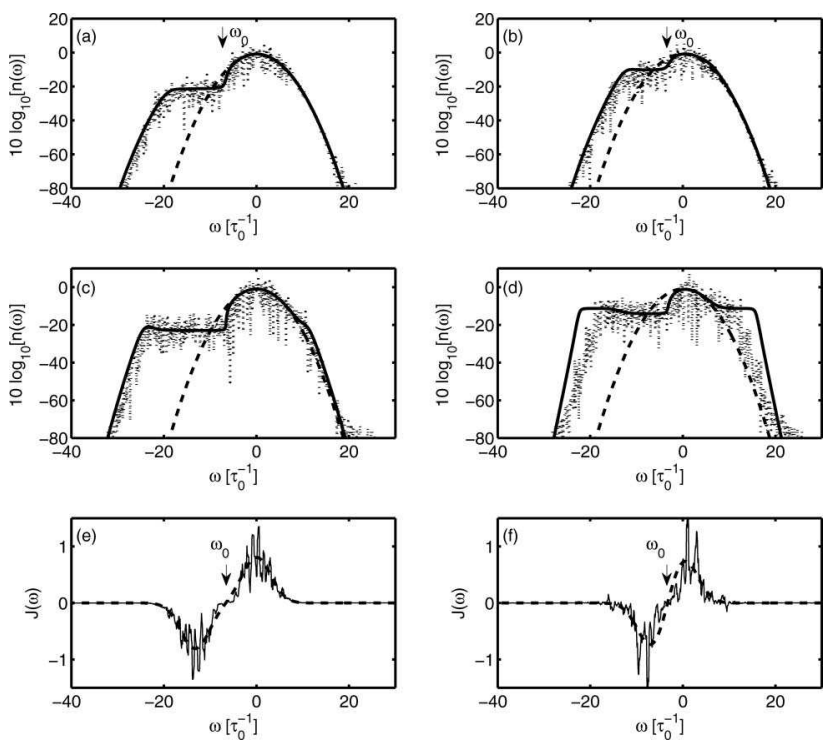

Fig. 1. First stage of the spectral evolution obtained by integrating numerically the NLSE (1) (dotted curve) and the kinetic Eq. (3) (solid curve) for $\alpha=0.05$ (left column) and $\alpha=0.1$ (right column): (a), (b) at $z=30$; (c), (d) at $z=2500$ $(\sigma=1)$. The dashed curve shows the initial condition $(z=0)$. (e), (f) Corresponding invariant $J(\omega)$ determined from the initial condition (dashed curve) and obtained by solving the NLSE (1) at $z=2500$ (solid curve). 

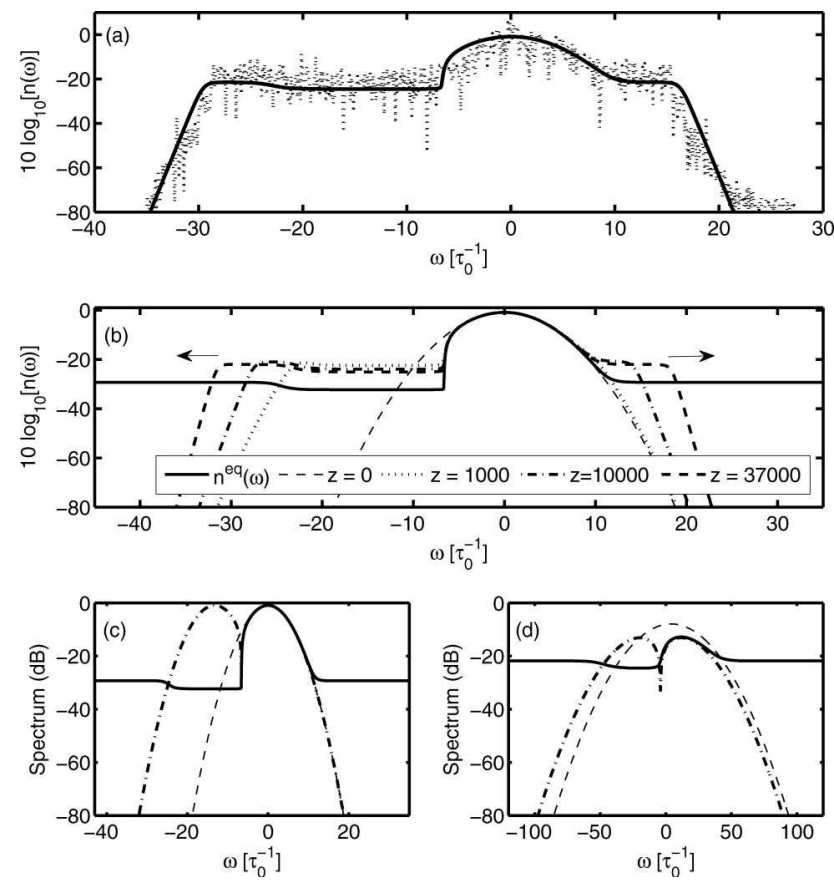

Fig. 2. (a) Second stage of the spectral evolution obtained by integrating numerically the NLSE (1) (dotted curve) and the kinetic Eq. (3) (solid curve) at $z=20000$ and (b) for different propagation lengths: a constant spectral pedestal emerges in the tails of the spectrum $(\alpha=0.05, \sigma=1)$. The spectrum slowly relaxes toward the equilibrium state $n^{\mathrm{eq}}(\omega)$ given by Eq. (4) (solid curve). (c), (d) Equilibrium spectrum $n^{\mathrm{eq}}(\omega)$ [Eq. (4)] (solid curve), initial condition (dashed curve), and corresponding invariant $\left|J_{\omega}\right|$ (dashed-dotted curve) for (c) $\alpha=0.05$ and (d) $\alpha=0.08$ : the invariant $J_{\omega}$ determines the essential properties of $n^{\mathrm{eq}}(\omega)$.

numerically the NLSE (1). The initial condition is a partially coherent wave with a Gaussian spectrum and random spectral phases (dashed curve), i.e., $u(z=0, t)$ is of zero mean and obeys stationary Gaussian statistics. We remark that the high-frequency tail of the spectrum does not exhibit any significant spectral broadening, whereas, in the lowfrequency part (anomalous dispersion), a broad spectral shoulder emerges, which is then preserved for long propagation lengths [Figs. 1(a) and 1(c)]. In this example, the initial spectrum lies essentially in the normal dispersion regime. We remark, however, that a similar spectral deformation is obtained by pumping in the anomalous disper$\operatorname{sion}(\sigma=-1)$, although, in this case, the deformation of the spectrum is reversed, i.e., the spectral shoulder emerges in normal dispersion. Note that such a reversed deformation of the spectrum also occurs by changing the sign of $\alpha$ (keeping $\sigma=1)$. The system is not sensitive to the sign of the dispersion coefficient $(\sigma)$, because we deal here with the weakly nonlinear regime, $U / E \ll 1$, which is known to suppress the modulational instability [13], and the generation of robust coherent soliton structures $[9,10,14]$. Another consequence of $U / E \ll 1$ is that the field statistics do not need to be Gaussian initially: linear dispersive effects dominate the interaction and bring the system to a state of stationary Gaussian statistics (a feature that we have verified). Note that the simulation of Fig. 1 ( $\alpha=$ 0.05 ) typically corresponds to light propagation in a photonic crystal fiber with $\gamma=50 \times 10^{-3} \mathrm{~W}^{-1} \mathrm{~m}^{-1}, \beta_{2}=$ $50 \times 10^{-27} \mathrm{~s}^{2} / \mathrm{m}$, and $\beta_{3}=27 \times 10^{-41} \mathrm{~s}^{3} / \mathrm{m}$. A thousand of nonlinear propagation lengths $L_{0}$ would then correspond to a fiber length of $50 \mathrm{~m}$ and an average power of $P=$ $400 \mathrm{~W}$ (neglecting the influence of the dissipative Raman effect).

A physical insight into this problem is obtained by making use of the WT theory. The kinetic wave theory is essentially based on the random phase approximation, which is known to break the formal reversibility of the NLSE. One may thus derive an irreversible kinetic equation describing the evolution of the averaged spectrum of the field, $n(z, \omega)=\left\langle|\tilde{u}|^{2}(z, \omega)\right\rangle[\underline{9-14}]$. It reads $\partial_{z} n(z, \omega)$ $=\operatorname{Coll}[n]$, where the collision term is $\operatorname{Coll}[n]=$ $\int d \omega_{1} d \omega_{2} d \omega_{3} W n(\omega) n\left(\omega_{1}\right) n\left(\omega_{2}\right) n\left(\omega_{3}\right)\left[n^{-1}(\omega)+n^{-1}\left(\omega_{1}\right)\right.$ $\left.-n^{-1}\left(\omega_{2}\right)-n^{-1}\left(\omega_{3}\right)\right]$, with $\quad W=\frac{1}{\pi} \delta\left(\omega+\omega_{1}-\omega_{2}-\omega_{3}\right)$ $\delta\left[k(\omega)+k\left(\omega_{1}\right)-k\left(\omega_{2}\right)-k\left(\omega_{3}\right)\right]$. Two integrals of Coll $[n]$ may be computed exactly owing to the $\delta$ functions in $W$. One obtains $\partial_{z} n(\omega, z)=\frac{1}{3 \pi \mid \alpha} \int \mathcal{G}[n] d \omega_{1}$, where the functional $\mathcal{G}[n]$ exhibits a remarkable property: it is invariant under the substitution $\omega \rightarrow \bar{\omega}=q-\omega$, i.e., $\mathcal{G}[n(\bar{\omega})]=$ $\mathcal{G}[n(\omega)]$, where $q=-2 \sigma / 3 \alpha=2 \omega_{0}$. This peculiar property implies $\partial_{z} n(\omega, z)=\partial_{z} n(\bar{\omega}, z)$, which thus reveals the existence of the following local invariant:

$$
J(\omega)=n(\omega, z)-n(q-\omega, z) .
$$

This invariant is local in the sense that it is verified for each frequency $\omega$ individually, $\partial_{z} J(\omega)=0$. It means that the subtraction of the spectrum by the reverse of itself translated by $q=2 \omega_{0}$ remains invariant during the whole evolution of the wave. The invariant of Eq. (2) finds its origin in the degenerate resonance of the phase-matching conditions: a pair of frequencies $(\omega, q-\omega)$ may resonate with any pair of frequencies $\left(\omega^{\prime}, q-\omega^{\prime}\right)$, because $k(\omega)+k(q-$ $\omega)=\sigma q^{2} / 3$ does not depend on $\omega$. Making use of $J(\omega)$, we obtain the following kinetic equation governing the evolution of the averaged spectrum of the wave:

$$
\partial_{z} n(\omega, z)=\frac{1}{3 \pi|\alpha|} \int \frac{\mathcal{F}[n, J]}{\left|\omega-\omega_{1}\right|\left|\omega+\omega_{1}-q\right|} d \omega_{1},
$$

with $\mathcal{F}[n, J]=n_{\omega_{1}}\left(n_{\omega}-J_{\omega}\right)\left(n_{\omega_{1}}-J_{\omega_{1}}\right)+n_{\omega} n_{\omega_{1}}\left(n_{\omega_{1}}-J_{\omega_{1}}\right)$ $-n_{\omega}\left(n_{\omega}-J_{\omega}\right)\left(n_{\omega_{1}}-J_{\omega_{1}}\right)-n_{\omega_{1}} n_{\omega}\left(n_{\omega}-J_{\omega}\right)$, where $J_{\omega}$ stands for $J(\omega)$. This kinetic equation has been integrated numerically [see Figs. 1(a) and 1(c), solid curve]. The invariant in Eq. (3) is fixed by the initial spectrum of the field, $J_{\omega}=n_{\omega}(z=0)-n_{q-\omega}(z=0)$. Let us stress the quantitative agreement (down to $-80 \mathrm{~dB}$ ) with the numerical simulations of the NLSE without any adjustable parameter, a feature that is corroborated by the fact that the NLSE conserves (in average) the invariant $J_{\omega}$ [see Fig. 1(e)]. This good agreement stems from the fact that we considered the weakly nonlinear regime, i.e., $|U / E| \sim$ 0.1 in Fig. 1. Note, however, that, as the parameter $\alpha$ increases, a significant amount of power evolves in the neighborhood of the zero dispersion frequency where nonlinear effects dominate, so that the criterion $|U / E| \ll$ 1 is not verified for $\omega \sim \omega_{0}$. We thus observed a discrepancy between the kinetic Eq. (3) and the NLSE (1) (see Fig. 1, right column), although the invariant $J_{\omega}$ is still preserved by the NLSE [Fig. 1(f)] 
The analysis of the invariant $J_{\omega}$ provides a simple qualitative interpretation of the asymmetric deformation of the spectrum discussed in Fig. 1. For this purpose, one should consider that the natural tendency of a nonlinear wave is to generate new frequency components in the tails of its spectrum, which leads to a lowering of the central part of the spectrum. Here, the lowering of the spectrum is constrained by the existence of the invariant $J_{\omega}$, because $n_{\omega}=J_{\omega}+n_{q-\omega} \geq J_{\omega}\left(n_{q-\omega} \geq 0\right)$. It turns out that the spectrum tends to approach the spectral profile of $J_{\omega}$ for those frequencies verifying $J_{\omega} \geq 0$, i.e., $n_{\omega} \simeq J_{\omega}$ for $\omega \geq q / 2=\omega_{0}$ [also see Fig. 2(d)]. For the frequencies $\omega \leq \omega_{0}$, the above expression reads $n_{q-\omega} \simeq-J_{\omega}$ (because $J_{q-\omega}=-J_{\omega}$ ), which leads to $n_{\omega}=J_{\omega}+n_{q-\omega} \simeq 0$. In summary, in the normal dispersion regime $\left(\omega \geq \omega_{0}\right)$, the spectrum evolves toward $J_{\omega}$, while, in the anomalous dispersion regime $\left(\omega \leq \omega_{0}\right)$, the spectral amplitude is small and almost constant. This provides a simple interpretation of the emergence of the spectral shoulder discussed in Fig. 1, which characterizes the first stage of the spectral evolution.

Next the system enters into a second stage, in which one observes the emergence of a constant spectral pedestal in the far tails of the wave spectrum [see Figs. 2(a) and 2(b)]. The spectral pedestal progressively emerges as a result of two fronts that propagate in opposite directions in frequency space, and symmetrically with respect to the zero dispersion frequency, $\omega_{0}=q / 2$. Such a symmetric front propagation is a consequence of the degenerate resonance discussed above through $J_{\omega}$, simply because the pairs of frequencies $\left(\omega_{j}, q-\omega_{j}\right)$ involved in the conversion $\left(\omega_{1}, q-\omega_{1}\right) \rightarrow\left(\omega_{2}, q-\omega_{2}\right)$ are always symmetric with respect to $\omega_{0}$. It turns out that the two fronts propagate with the same velocity in frequency space, although they are asymmetric with respect to the carrier frequency of the wave, i.e., $\omega=0$.

The unexpected formation of a constant spectral pedestal is, in fact, a consequence of the natural relaxation of the wave toward its equilibrium state. Indeed, the kinetic Eq. (3) exhibits an $H$ theorem of entropy growth, $\partial_{z} S \geq 0$, with the nonequilibrium entropy $S(z)=\int \log \left(n_{\omega}\right) d \omega$. Equation (3) also conserves the power $N$, the energy $E$, and the momentum $M$. The equilibrium spectrum $n^{\mathrm{eq}}(\omega)$ that realizes the maximum of $\mathcal{S}[n]$ reads

$$
n^{\mathrm{eq}}(\omega)=J_{\omega} / 2+\left[1+\sqrt{1+\lambda^{2} J_{\omega}^{2} / 4}\right] / \lambda .
$$

The parameter $\lambda$ is determined from the conservation of the power, $N=\int n^{\mathrm{eq}}(\omega) d \omega=\int n(\omega, z=0) d \omega$, while the conservations of $E$ and $M$ are implicitly verified through the invariant $J_{\omega}$. This equilibrium distribution is of a different nature than the conventional RJ equilibrium distribution, as discussed in detail in [15]. A peculiar property of $n^{\mathrm{eq}}(\omega)$ is precisely the fact that it exhibits a constant spectral pedestal, $n^{\mathrm{eq}}(\omega) \rightarrow 2 / \lambda$ for $|\omega| \gg|q|$. We remark that the equilibrium spectrum $n^{\mathrm{eq}}(\omega)$ exhibits a lateral dip in the anomalous dispersion regime, whose frequency precisely corresponds to the frequency of the minimum of $J(\omega)$ [see Figs. 2(c) and 2(d)].

In summary, we developed a kinetic theory of incoherent light propagation in the presence of TOD. It reveals the existence of an invariant $J_{\omega}$ that determines the essential properties of the spectral evolution of the wave.

This research was supported by the Agence Nationale de la Recherche (ANR-COSTUME 08-SYSC-004-03).

\section{References}

1. B. Barviau, S. Randoux, and P. Suret, Opt. Lett. 31 1696 (2006).

2. A. Mussot, A. Kudlinski, M. Kolobov, E. Louvergneaux, M. Douay, and M. Taki, Opt. Express 17, 17010 (2009).

3. S. Babin, V. Karalekas, E. Podivilov, V. Mezentsev, P. Harper, J. Ania-Castañón, and S. Turitsyn, Phys. Rev. A 77, 033803 (2008).

4. D. Dylov and J. Fleischer, Phys. Rev. A 78, 061804 (2008).

5. U. Bortolozzo, J. Laurie, S. Nazarenko, and S. Residori, J. Opt. Soc. Am. B 26, 2280 (2009).

6. A. Fratalocchi, C. Conti, G. Ruocco, and S. Trillo, Phys. Rev. Lett. 101, 044101 (2008).

7. Y. Silberberg, Y. Lahini, E. Small, and R. Morandotti, Phys. Rev. Lett. 102, 233904 (2009).

8. A. Picozzi, S. Pitois, and G. Millot, Phys. Rev. Lett. 101, 093901 (2008).

9. B. Barviau, B. Kibler, S. Coen, and A. Picozzi, Opt. Lett. 33, 2833 (2008).

10. B. Barviau, B. Kibler, A. Kudlinski, A. Mussot, G. Millot, and A. Picozzi, Opt. Express 17, 7392 (2009).

11. A. Picozzi, Opt. Express 16, 17171 (2008).

12. S. Lagrange, H. R. Jauslin, and A. Picozzi, Europhys. Lett. 79, 64001 (2007).

13. A. Picozzi, Opt. Express 15, 9063 (2007).

14. V. E. Zakharov, F. Dias, and A. Pushkarev, Phys. Rep. 398, 1 (2004).

15. P. Suret, S. Randoux, H. R. Jauslin, and A. Picozzi, Phys. Rev. Lett. 104, 054101 (2010). 